\section{Estimating visual acuity by character counting using the Snellen visual acuity chart}

\section{Materials and methods}

After institutional review board approval and informed consent, 35 healthy volunteers (14 male, 21 female; mean age 26 years, range $20-53$ years) were recruited. The inclusion criteria were: age 18 years or older, no ophthalmic diseases other than refractive error, and right eye visual acuity of 20/30 or better with current correction. Subjects, such as ophthalmic technicians, familiar with the Snellen visual acuity chart were excluded.

The commonly used projected Snellen visual acuity chart was used at a test distance of $20 \mathrm{ft}$, simulated by reflecting the chart with a mirror on an opposite wall. This chart was chosen instead of more sophisticated balanced visual acuity charts based on log of minimal angle of resolution because this chart is widely used clinically and has a variable number of characters at different acuity levels. Visual acuity was measured in a standard manner as the smallest Snellen chart line that the subject could correctly identify the letters. Two errors were allowed for lines with five or more letters, one error on lines with four letters, and no errors were allowed for the two letters on the 20/200 line. Counting level was defined as the

\section{Introduction}

Most measures of visual acuity test one of the following: detection, recognition, resolution, or

\begin{abstract}
localization. ${ }^{1}$ Conventional testing with the Snellen visual acuity chart (Reichert 11180) measures letter recognition. Detection, measured by the ability to perceive the presence of, as opposed to identify, an object can also be tested with the Snellen chart by evaluating an individual's ability to count letters and has been suggested as a method to estimate visual acuity in patients with nonorganic visual loss. ${ }^{2}$ However, to our knowledge, this is the first evaluation of the exact correlation between conventional visual acuity measurements and character counting using the Snellen acuity chart.

Purpose This study examines visual acuity
\end{abstract} loss; malingering; nonorganic visual loss; visual acuity

\author{
financial interest in the \\ material presented. \\ part from a Research to \\ Prevent Blindness \\ departmental grant. \\ The authors have no \\ ${ }^{1}$ Bascom \\ University of Miami School \\ Accepted: 15 March 2004 \\ Published online: 28 May
} (1) 
Table 1 Corresponding Snellen visual acuities for each level of counting acuity

\begin{tabular}{lccccc}
\hline $\begin{array}{l}\text { Counting } \\
\text { acuity }\end{array}$ & Number & $\begin{array}{l}\text { Median corresponding } \\
\text { snellen visual acuity }\end{array}$ & $\begin{array}{l}\text { Minimum corresponding } \\
\text { Snellen visual acuity }\end{array}$ & $\begin{array}{l}\text { Maximum corresponding } \\
\text { Snellen visual acuity }\end{array}$ & $\begin{array}{l}\text { Upper 95th percentile } \\
\text { corresponding Snellen } \\
\text { visual acuity }\end{array}$ \\
\hline $20 / 10$ & 17 & $20 / 25$ & $20 / 15$ & $20 / 70$ & $20 / 30$ \\
$20 / 15$ & 24 & $20 / 30$ & $20 / 20$ & $20 / 70$ & $20 / 50$ \\
$20 / 20$ & 31 & $20 / 40$ & $20 / 30$ & $20 / 200$ & $20 / 80$ \\
$20 / 25$ & 14 & $20 / 50$ & $20 / 20$ & $20 / 80$ & $20 / 80$ \\
$20 / 30$ & 9 & $20 / 80$ & $20 / 40$ & $20 / 200$ & $20 / 200$ \\
$20 / 40$ & 24 & $20 / 80$ & $20 / 50$ & $20 / 200$ & $20 / 200$ \\
$20 / 50$ & 16 & $20 / 100$ & $20 / 70$ & $20 / 200$ & $20 / 200$ \\
$20 / 60$ & 17 & $20 / 200$ & $20 / 80$ & & 200 \\
\hline
\end{tabular}

smallest Snellen chart line that the subject could correctly count the number of letters without error. The 20/400 line was not used because it is the only line represented by a single letter, and the $20 / 400$ ' $\mathrm{E}^{\prime}$ was readily recognizable and detectable. Using the phoropter, the left eye of each subject was occluded and the right eye was fogged several times with spherical plus lenses of different powers. Visual acuity and counting level were assessed at each level of fogging. To limit any learning effect, each subject was evaluated at no more than five levels of fogging.

\section{Results}

Table 1 summarizes the data and Figure 1 plots visual acuity against the corresponding counting levels. The number of subjects who contributed to each counting level varied as a result of random fogging. For counting levels from 20/10 to 20/60, the size of the letters of the median corresponding visual acuity was approximately twice the size of the letters of the counting level. For example, $50 \%$ of the subjects who were able to count the number of letters on the 20/15 line had a corresponding fogged visual acuity of $20 / 30$ or better, and $50 \%$ of the subjects who were able to count the number of characters on the 20/20 line had a corresponding visual acuity of $20 / 40$ or better. For a given counting level, the upper 95 th percentile Snellen visual acuity is the visual acuity that $95 \%$ of our sample saw equal or better than. For instance, based on the upper 95th percentile corresponding Snellen visual acuity, a subject with a counting level of $20 / 15$ is very likely (ie $95 \%$ chance) to have a visual acuity equal to or better than 20/50, and a subject with a counting level of $20 / 20$ is very likely to have a visual acuity equal to or better than $20 / 80$.

The correlation between counting level and visual acuity was close for counting levels of 20/25 or better. For each of these counting levels, the upper 95th percentile cutoff visual acuity was slightly greater than

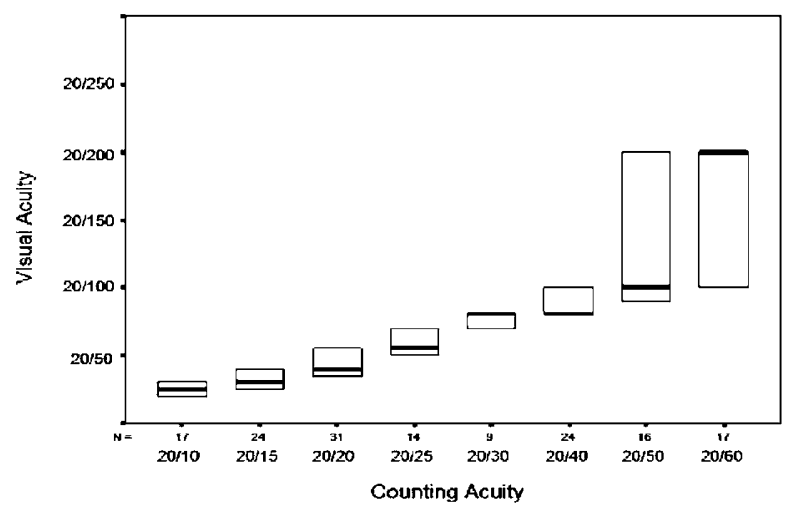

Figure 1 Relationship between character counting and visual acuity on the Snellen visual acuity chart (Reichert 11180). Character counting levels are plotted against the corresponding visual acuity for the pooled data from 35 subjects. The number of subjects $(n)$ for each counting level varied as a result of random fogging. A close correlation was observed between counting acuity and visual acuity (Spearman's nonparametic $r=0.89$, $P<0.0001)$.

the corresponding median visual acuity. In contrast, for all counting levels of 20/30-20/60, the corresponding upper $95 \%$ cutoff visual acuity was $20 / 200$. Thus, at these counting levels the predicted visual acuity is less precise and all that can be concluded with certainty is that the patient has a visual acuity equal to or better than 20/200. When fogged to a counting level of $20 / 70$, some test subjects had a corresponding visual acuity of 20/400 or worse. This is beyond the visual acuity level that this study was designed to evaluate; therefore, data for counting levels of 20/70 or worse were not consistently collected and are not included.

\section{Discussion}

Although the Snellen chart is designed to test letter recognition, our data suggest that characters counting 
roughly estimates visual acuity, and may be useful with patients who are unable to be tested with standard techniques, such as those with nonorganic visual loss.

The size of the letters of the corresponding visual acuity was most often twice the size of the counting level letters. However, the upper 95th percentile corresponding visual acuity probably provides the most useful information, because in most instances an underestimation is preferred to an overestimation of visual acuity. Based on the upper 95th percentile corresponding visual acuity, a subject with a documented counting level of 20/10 is very likely (ie $95 \%$ chance) to have a visual acuity equal to or better than 20/30, and counting levels of 20/15, 20/ 20 , and $20 / 25$ are very likely to estimate a visual acuity of 20/50, 20/80, and 20/80, respectively. However, at counting levels, $20 / 30-20 / 60$, all that can be concluded is that a subject has a visual acuity equal to or better than $20 / 200$. Nevertheless, a documented counting level in this range (20/30-20/60) may still be valuable in establishing visual acuity at least 20/200 in a subject suspected to be malingering with an alleged visual acuity of 20/400 or worse.

The loss of the ability to predict precisely subjects' visual acuity from their counting level with increasing degrees of fogging is likely related to the Snellen chart design. For counting levels including and between 20/30 and $20 / 60$, the corresponding approximate 95 th

percentile cutoff visual acuity was the same: 20/200. At these counting levels, the actual visual acuity of many individuals and therefore the upper $95 \%$ cutoff acuity likely lies somewhere between 20/100 and 20/200.

However, due to limitations of the Snellen acuity chart (ie the lack of visual acuity lines between 20/100 and 20/ 200), such individuals would have a measured visual acuity of 20/200. Therefore, the discrepancy between the predictability of visual acuity at different counting levels is likely in part a function of the Snellen chart. A closer correlation might be found in the 20/30-20/60 counting acuity range with a logmar chart, where increases in visual angle are uniformly graduated.

Potential limitations of this study include the fact that subjects with ocular conditions other than induced refractive error were not evaluated. Certain organic diseases may affect standard visual acuity and counting acuity to different degrees. Possible examples include hemianopsia where due to the loss of half the characters, letters cannot be correctly identified but still counted. A similar situation can be envisioned with metamorphopsia due to macular disease. Therefore, as with all tests for functional visual loss, counting acuity should be interpreted in the context of associated findings. Moreover, no comparison was made with conventional methods of testing alleged malingerers, such as neutralizing cylinders. The proportion of patients with functional visual loss that counting acuity proves to be a beneficial addition to established methods remains to be determined. Although counting responses that are inconsistent with Snellen acuity is helpful in establishing functional visual loss, counting acuity should not be considered a substitute for standard Snellen acuity. The nature of the errors on standard Snellen chart testing is likely more informative. Lastly, character counting is less useful in subjects familiar with the Snellen chart. These limitations should be considered when interpreting results. However, there are several advantages to using letter counting including being convenient and quick, due to the availability of Snellen charts and the simplicity of testing. Moreover, patients with nonorganic visual loss, who often undergo multiple examinations, are likely unfamiliar with character counting and may not readily recognize the intent of the test.

In short, our data suggest that character counting testing can provide a rough visual acuity estimate. It may be useful when applied to patients who cannot be accurately tested with standard visual acuity assessment techniques including those suspected to have nonorganic visual loss.

\section{References}

1 Riggs LA. Visual acuity. In: Grahm CH (ed). Vision and Visual Perception. Wiley: New York, NY, 1966, pp 321-349.

2 Kramer KK, La Piana FG, Appleton B. Ocular malingering and hysteria: diagnosis and management. Surv Ophthalmol 1979; 24: 89-96. 